ISSN 1112-9867

Available online at $\quad$ http://www.jfas.info

\title{
REBA EVALUATION ON GARAGE WORKER: A CASE STUDY
}

\author{
A. Shukriah*, M. D. Baba, and A.G. Jaharah \\ Faculty of Engineering and Built Environment, Universiti Kebangsaan Malaysia, 43600, \\ Bangi, Selangor, Malaysia
}

Published online: 17 October 2017

\begin{abstract}
Garage workers always involve with awkward posture as their task being carried out manually in most small scale vehicle maintenance industries. Therefore it is crucial to identify the problems causing awkward posture because it can result to the development of Musculoskeletal Disorder Symptoms (MSDs), which may gone unnoticed and might cause higher compensation cost later. Postural analysis tool using Rapid Entire Body Assessment (REBA) were applied and conducted on nine workers engaging in maintaining three types of vehicle namely car, Multi-purpose vehicle and bus. The evaluation of working postures in different activities is carried out using worksheet of scoring system. Result shows that most of the tasks involved awkward posture and most at high risk level. There is lack of ergonomic awareness and knowledge which contribute more towards high risk of MSDs problem.
\end{abstract}

Keywords: garage worker; assessment tools; musculoskeletal disorder; REBA; ergonomic.

Author Correspondence, e-mail: shuk.kye@gmail.com

doi: http://dx.doi.org/10.4314/jfas.v9i5s.74 


\section{INTRODUCTION}

\subsection{Garage worker and their task}

Garage worker provoke repetitive, static and awkward motion that are a common source of serious Musculoskeletal disorder. The involvement in awkward posture frequently and for a prolonged period of time is due to handling wide range of task from inspecting, diagnosing, repairing and replacing in order to maintain and upgrade vehicles. Fatigue and muscle strain can accumulate when unnatural or awkward posture and forceful exertions are involved. The postural targeting method REBA is used to estimate the risk of work-related entire body disorder. It is an assessment of quick and systematic to evaluate complete body posture, forceful exertion, type of movement or action, repetition and coupling. This study is to detect whether the daily vehicle maintenance tasks carried out by the garage worker are in high risk resulting to body disorder.

\subsection{Ergonomic intervention}

Ergonomic is a scientific discipline of understanding interaction between human and other systems which applies theory, data, method and principle to optimize those performances [1]. Regarding on working smarter, waste elimination, and maintaining system, the ergonomic impacts had influence within the industry field which is to optimize the interface between human and systems. There are several principles of ergonomic that have been emphasized which are work in neutral posture, reduce excessive force and motions, keep everything in easy reach, work at proper heights, minimized fatigue and static load, minimize pressure points, provide clearance, move, exercise and stretch, maintain a comfortable environment, make displays and controls understandable, and improve work organization [2][3][4]. Ergonomic risks factor detection in workplace as an essential step in correcting hazard as well as improving worker safety. Workers suffering from ergonomic related injuries required more time off the job compare with other types of workplace injuries [5]. Workers play an essential role in helping the company to create a workplace in which the employee can work productively and comfortably. The most talked about physical problem caused by poor ergonomic is Musculoskeletal Disorder (MSDs). These types of illness develop over long periods of time is painful and will affect the body condition. Because of the repetitive and awkward movement that mostly the workers spend hours in engaging their work, the 
possibility and risk to expose to MSDs become higher. Furthermore, studies in ergonomic among industry are inadequate due to lack of time, the management commitment as well as work pressure $[6][7][8][9][10]$.

\section{RESULTS AND DISCUSSION}

The nine workers were selected for this study. They worked on different types of vehicle (three types) with average age from 23-38 years old, with the height $165-171 \mathrm{~cm}$ and normal body weight with daily work lasting around eight or more hours. These workers frequently involve in bending, kneeling and sustained postures. Observing video was recorded while maintenance task being carried out and had recorded their posture and movement. The snapshot of each awkward posture were used and analyzed by filling the worksheet score in REBA. Table 1 showed the score evaluation from the worksheet. Fig. 1 illustrated the REBA score evaluate from the task each garage worker did, at the time of observation and all of the task present are high risk according to the evaluation REBA scoring. A1.A2, and A3, represented the garage worker, the three types of vehicle represent three garage workers with three different task. Task one is clean the car valve, task two replace car tire absorber and task three replace fan. From the video observation most of the workers faced with awkward posture, which can be listed as; head/body turning, bending, squat, reaching, flexing, lift, push and pull a weight load. Other concerns that are discovered are such as working static in prolonged time, working in narrow space and the use of non-adjustable chair.

Table 1. Scoring

\begin{tabular}{ll}
\hline Score & \multicolumn{1}{c}{ Risk } \\
\hline 1 & Negligible risk \\
$1-3$ & Low risk. Change may be needed \\
$4-7$ & Medium risk. Further investigation. Change soon \\
$8-10$ & High risk. Investigation and implement change \\
$11+$ & Very high risk. Implement change \\
\hline
\end{tabular}

Please use this paragraph to type the table footer(s) if any. 


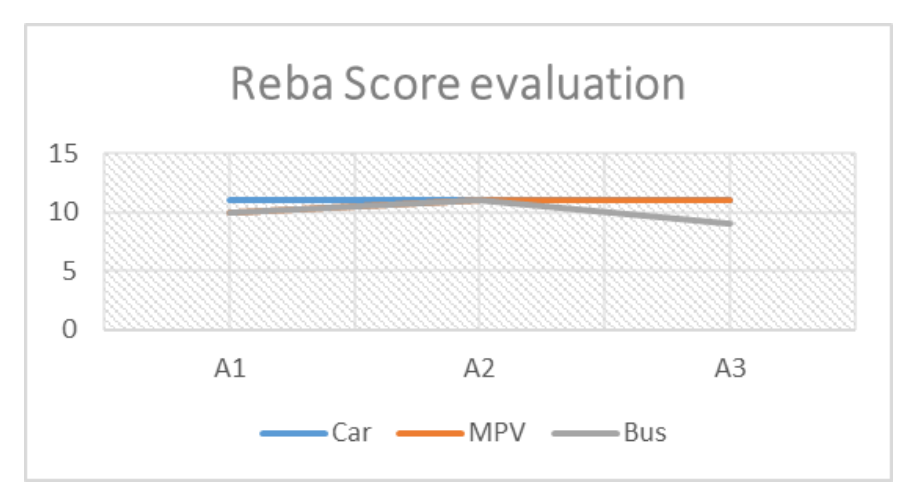

Fig.1. Reba score evaluation

\section{EXPERIMENTAL}

How the REBA worksheets work; there are two major parts to assess which are A; neck, trunk and leg analysis and B; arm and wrist analysis. Each of the parts consists of step continuing from step 1 to step 6 in part A and step 7 to step 13 in part B. There is a table matric use to align the score evaluated from each neck, trunk, and leg score. Activity, coupling and load/ force are three other item that exist during evaluation and are represented in Table 2, 3 and 4. Fig. 2 is the example of overall worksheets assessment score for A1, task 1, and vehicle type car.

Table 2. Activity Score

\begin{tabular}{cl}
\hline Activity & \multicolumn{1}{c}{ Explanation } \\
\hline+1 & 1 or more body parts are held for longer than 1 minute (static) \\
+1 & Repeated small range actions (more than $4 \mathrm{x}$ per minute) \\
+1 & Action causes rapid large range change in postures or unstable base \\
\hline
\end{tabular}

Table 3. Coupling

\begin{tabular}{ll}
\hline \multicolumn{1}{c}{ Activity } & \multicolumn{1}{c}{ Explanation } \\
\hline 0 good & Well-fitting handle \& a mid range power grip \\
1 fair & Hand hold acceptable but not ideal, or coupling is acceptable via \\
& another part of the body \\
2 poor & Hand hold not acceptable although possible \\
3 unacceptable & Awkward, unsafe grip, no handle: coupling unacceptable using \\
& other parts of the body \\
\hline
\end{tabular}


Table 4. Load / Force

\begin{tabular}{cl}
\hline Activity & \multicolumn{1}{c}{ Explanation } \\
\hline 0 & $<5 \mathrm{~kg}$ \\
1 & $5-10 \mathrm{~kg}$ \\
2 & $>10 \mathrm{~kg}$ \\
& Add +1 for shock or rapid build \\
\hline
\end{tabular}
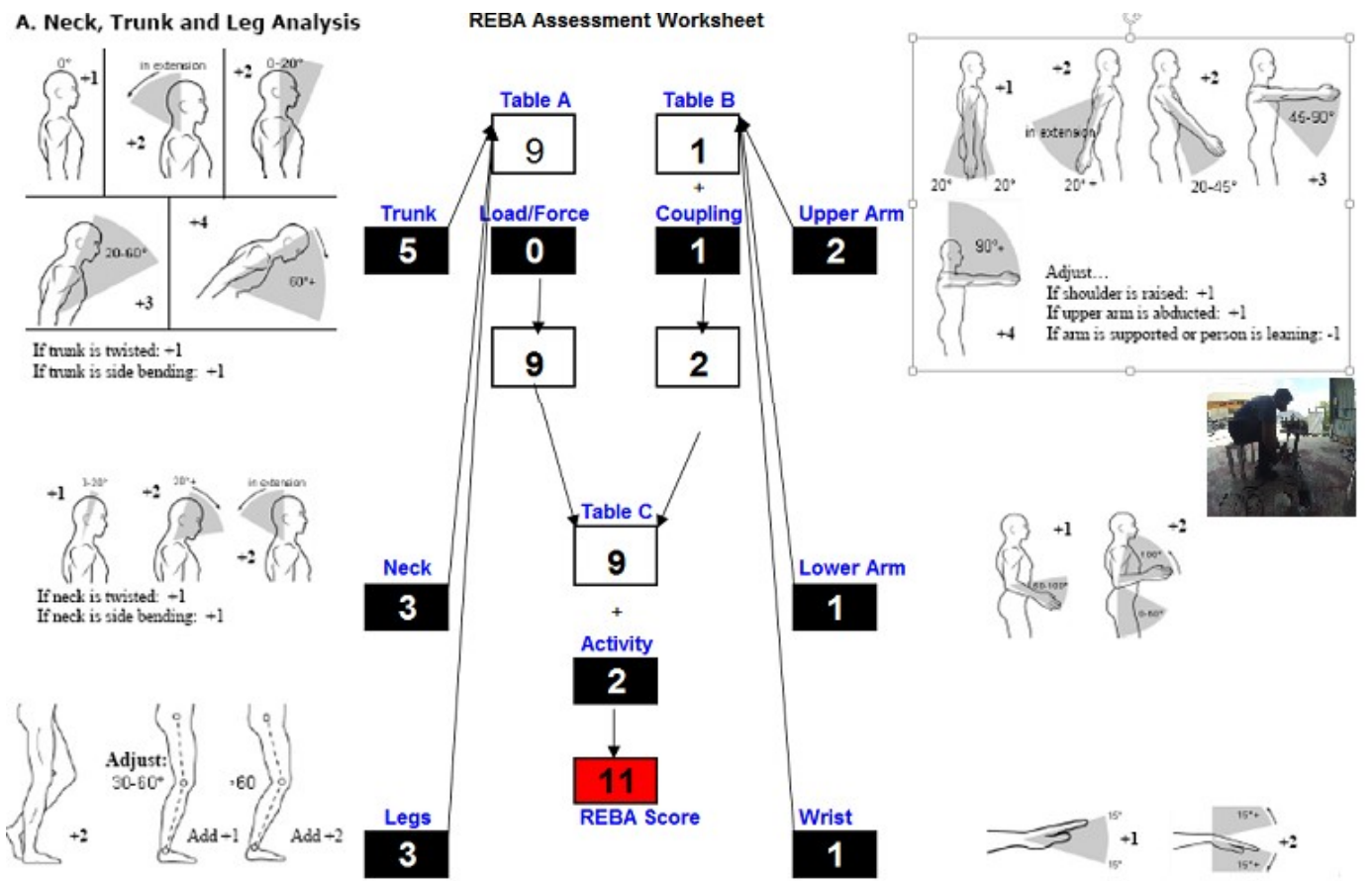

Fig.2. Overall assessment score for A1, task 1, vehicle type car

\section{CONCLUSION}

Most of the participants feel pain, ache and discomfort around their body area; neck, shoulder, elbow, wrist/hand, upper back, lower back, hip/thigh, and knees. This study used the ergonomic assessment sheets to show that most of the garage workers have high risk in developing MSDs. The important element in understanding the ergonomic interventions requirement came from several contributing factors that can be listed as; the organization culture stretch on health and safety, the involvement from the management either from support, rewarding, recognition and motivation, knowledge about the ergonomic from worker as well as from the management. Ergonomics related to more than one branch of knowledge, 
and are a wide range of science and practical as a concept. The involvement of the workers as a team to discuss their workstation effectiveness is useful and will provide job satisfaction and safe workstation.

\section{REFERENCES}

[1] International Ergonomics Association. Definition of ergonomics, 2010

[2] MacLeod D. The ergonomics edge: Improving safety, quality, and productivity. New Jersey: John Wiley \& Sons, 1994

[3] MacLeod D. The ergonomics kit for general industry. USA: CRC Press, 2006,

[4] MacLeod D. The rules of work: a practical engineering guide to ergonomics. USA: CRC Press, 2012

[5] Bossen D, Landsman J, and Robbins S. System and method for optimally determining appropriate ergonomics for occupants of a workspace. United State Patent, 2010, 1-55.

[6] Cole D C, Theberge N, Dixon S M, Rivilis I, Neumann, W P, and Wells R. Reflecting on a program of participatory ergonomics interventions: A multiple case study.

Work, 2009, 34(2):161-178

[7] Driessen M T, Groenewoud K, Proper K I, Anema J R, Bongers P M, and van der Beek A J. What are possible barriers and facilitators to implementation of a participatory ergonomics programme? Implementation Science, 2010, 5(1):1-9

[8] Hengel K M O, Blatter B M, van der Molen H F, Bongers P M, and van der Beek A J. The effectiveness of a construction worksite prevention program on work ability, health, and sick leave: Results from a cluster randomized controlled trial. Scandinavian Journal of Work, Environment \& Health, 2013, 39(5):456-467

[9] Cantley L F, Taiwo O A, Galusha D, Barbour R, Slade M D, Tessier-Sherman B, and Cullen M R. Effect of systematic ergonomic hazard identification and control implementation on musculoskeletal disorder and injury risk. Scandinavian Journal of Work, Environment \& Health, 2014, 40(1):57-65

[10] Dale A M, Jaegers L, Welch L, Gardner B T, Buchholz B, Weaver N, Evanoff B A. Evaluation of a participatory ergonomics intervention in small commercial construction firms. American Journal of Industrial Medicine, 2016, 59(6):465-475 
How to cite this article:

Shukriah A, Baba M D, and Jaharah A G. REBA evaluation on garage worker: A case study. J. Fundam. Appl. Sci., 2017, 9(5S), 1080-1086. 